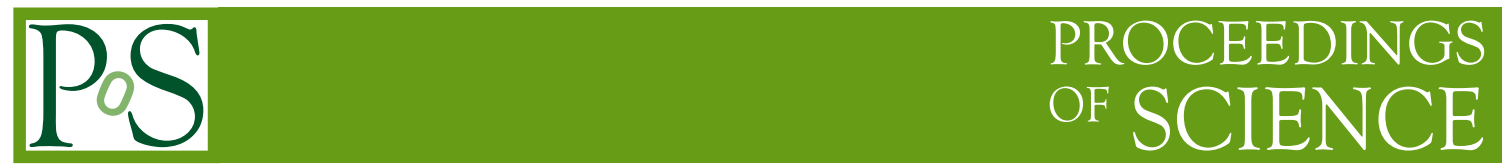

\title{
Jet measurements from CMS
}

\section{Debarati Roy}

Saha Institute of Nuclear Physics

On behalf of the CMS Collaboration

E-mail: debarati.roy@cern.ch

Recent results on jets measurements as well as jet properties and jet variables are presented. These studies illustrate the perturbative and non-perturbative aspects of QCD since jets are the most direct manifestations of these scenarios. The data samples used for these measurements were collected at proton-proton collisions with center-of-mass energy $(\sqrt{s})$ equal to $2.76,7$ and 8 $\mathrm{TeV}$ with the CMS detector at the LHC.

The European Physical Society Conference on High Energy Physics

22-29 July 2015

Vienna, Austria 


\section{Introduction}

Production of jets is one of the major processes in collisions at hadron colliders. Jets are collimated sprays of hadrons, which are essential to study the hard and non-perturbative QCD effects and the proton structure. In the LHC environment a detailed understanding of these phenomena is important, when measuring different physics processes. In these proceedings the presented results correspond to the recent measurements on inclusive jet cross sections, dijet azimuthal decorrelations, 3-jet mass cross sections and topological observables in multijet production, based on the analysis of data collected at $\sqrt{s}=2.76,7$ and $8 \mathrm{TeV}$ with the Compact Muon Solenoid (CMS) detector [1] at the CERN LHC.

\subsection{Inclusive Jet Production at $\sqrt{s}=8 \mathrm{TeV}$}

A measurement of the jet cross section as a function of the rapidity and the transverse momentum $\left(\mathrm{p}_{T}\right)$ of the jet is a sensitive quantity for probing the calculation of the hard parton interaction as well as the parton densities. The inclusive jet cross section measurement $[2,3]$ is performed in proton-proton (pp) collisions at $\sqrt{s}=8 \mathrm{TeV}$ data collected by the CMS detector, equivalent to an integrated luminosity of $5.8 \mathrm{pb}^{-1}$ for low $\mathrm{p}_{T}$ jets and an integrated luminosity of $10.71 \mathrm{fb}^{-1}$ for high $\mathrm{p}_{T}$ jets. The measurement is carried out using the anti- $\mathrm{k}_{T}$ clustering algorithm [4] with distance parameter $\mathrm{R}=0.7$ in the absolute rapidity range of $|\mathrm{y}|<4.7$ for jet transverse momenta $21<\mathrm{p}_{T}<2000 \mathrm{GeV}$. The measured jet cross-section is then compared to next-to-leading order (NLO) predictions using various PDF sets. Figure 1 (a) shows the comparison between data and NLO theory using the NNPDF2.1 PDF set and corrected for non-perturbative (NP) effects, while (b) presents the level of agreement along with the uncertainty bands. A nice agreement is observed between the data and theory through out the $\mathrm{p}_{T}$ and lyl ranges covered.

Figure 1: (a) Data to theory comparisons at $8 \mathrm{TeV}$ as a function of jet rapidity and jet transverse momentum (b) Data to theory ratio as a function of jet $\mathrm{p}_{T}$ along with their respective uncertainties



\subsection{Inclusive Jet Production at $\sqrt{s}=2.76 \mathrm{TeV}$}

CMS has delivered the measurement of the double differential jet cross section [5] as a function of jet $\mathrm{p}_{T}$ and lyl using pp collisions at $\sqrt{s}=2.76 \mathrm{TeV}$. The total integrated luminosity used is 5.43 $\mathrm{pb}^{-1}$, and the measurement covers a jet $\mathrm{p}_{T}$ range from 74 to $592 \mathrm{GeV}$ and a lyl range from 0.0 to 3.0. The measured jet cross section is then compared to NLO predictions using five different PDF sets and corrected for NP effects. The two adjacent distributions in Figure 2 exhibit two important characteristics : the left one shows a good agreement between the data and NLO predictions using the CT10 PDF set, while the right one indicates that mostly the level of agreement between data and theory lies within the ranges of the experimental and theoretical uncertainties.

The ratio of the inclusive jet cross sections at $\sqrt{s}=2.76 \mathrm{TeV}$ and $8 \mathrm{TeV}$ can be used to constrain PDFs since its precision improves due to a partial cancellation of uncertainties. In fact the theoretical prediction uncertainty band gets reduced as higher $\mathrm{p}_{T}$ range is reached (Figure 3). Therefore in order to quantify the agreement of the measurement with its theoretical prediction at these two center-of-mass energies this study provides significant useful information. It can be also seen that (Figure 3) among all theoretical predictions calculated with different PDF sets consistently closer agreement is found for the NNPDF3.0 PDF set.

Figure 2: (a) Data to theory comparisons at $2.76 \mathrm{TeV}$ as a function of jet rapidity and jet transverse momentum (b) Data to theory ratio as a function of jet $\mathrm{p}_{T}$ along with their respective uncertainties
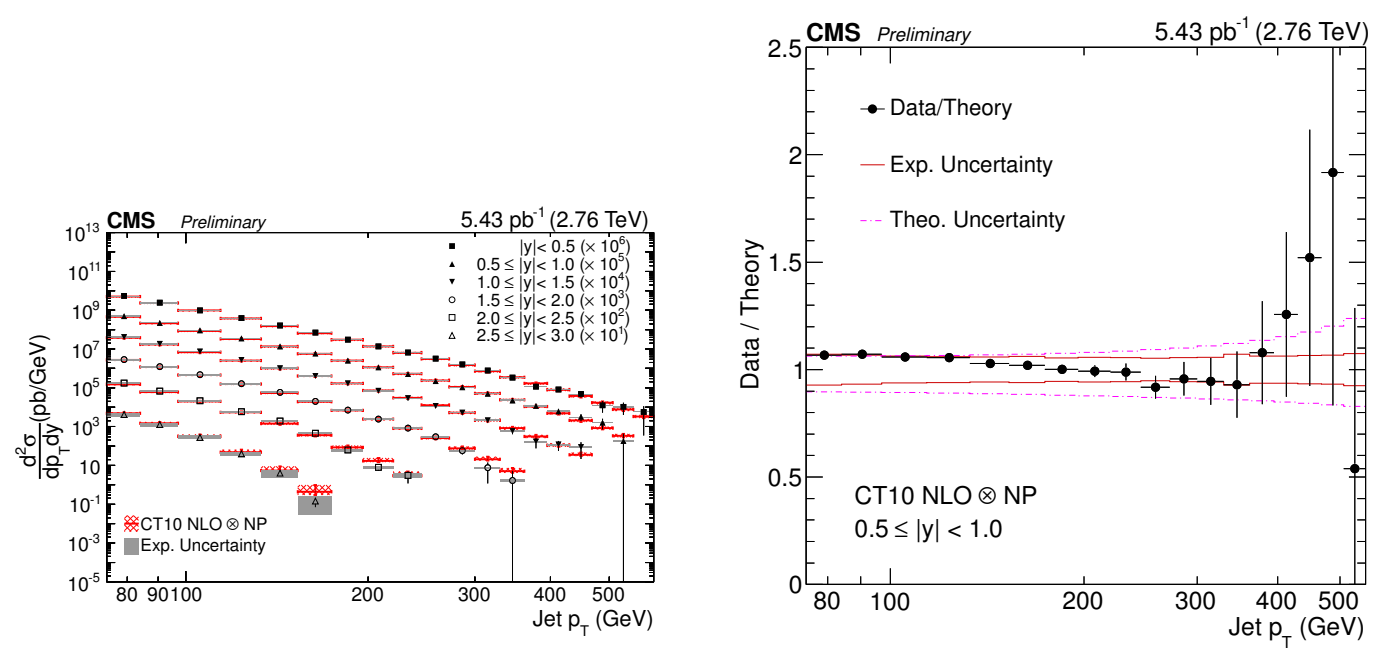

\subsection{Dijet azimuthal decorrelations at $\sqrt{s}=8 \mathrm{TeV}$}

The measurement of dijet azimuthal decorrelations between the two jets with the largest transverse momenta is measured [6] by CMS for seven regions of leading jet transverse momenta up to $2.2 \mathrm{TeV}$. The study is based on the full data set of pp collisions collected by CMS experiment at a center-of-mass energy of $8 \mathrm{TeV}$. The collected data correspond to an integrated luminosity of $19.7 \mathrm{fb}^{-1}$. The dijet azimuthal decorrelation is sensitive to the radiation of additional jets and probes the dynamics of multijet production. The results are compared to fixed-order predictions of 
Figure 3: The ratio of the inclusive jet cross sections at $\sqrt{s}=2.76 \mathrm{TeV}$ to $8 \mathrm{TeV}$
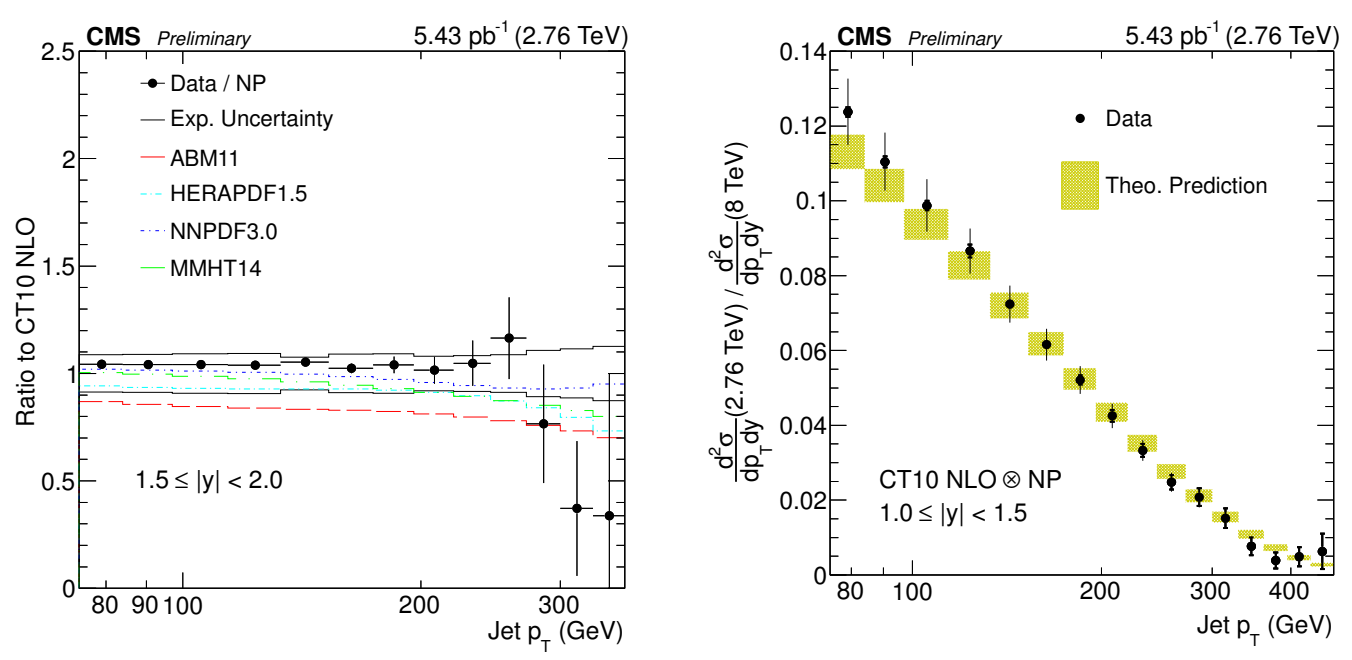

perturbative QCD and to various Monte Carlo event generators. Figure 4 shows the normalised differential dijet cross section as a function of azimuthal angular separation $\left(\Delta \phi_{\text {Dijet }}\right)$ between the two leading jets, together with the fixed order calculations in perturbative QCD using the CT10 PDF set. Theory calculations are available between $\pi / 2<\Delta \phi_{D i j e t}<\pi$ whereas the actual study covers a broader range $\left(0<\Delta \phi_{\text {Dijet }}<\pi\right)$. The LO calculation is available when $\Delta \phi_{\text {Dijet }}$ lies between $\pi / 2$ $<\Delta \phi_{\text {Dijet }}<2 \pi / 3$, while NLO predictions for 3 -jets are provided for $2 \pi / 3<\Delta \phi_{D i j e t}<\pi$. Figure 5 , on the left, shows the ratios of the normalised differential dijet cross sections in terms of $\Delta \phi_{\text {Dijet }}$ to the fixed-order perturbative QCD predictions using various PDF sets for all $\mathrm{p}_{T}^{\max }$ regions. The agreement between data and theory is reasonably good where the theory calculations are available at NLO level while they significantly diverge from each other for LO predictions. Figure 5, on the right, shows the ratios of various event generator predictions to the normalised differential dijet cross section in terms of $\Delta \phi_{\text {Dijet }}$. Among all generators MADGRAPH showered with PYTHIA6 performs the best.

\subsection{3-jet mass differential cross section at $\sqrt{s}=7 \mathrm{TeV}$}

CMS has delivered the measurement of the inclusive 3-jet mass differential cross section [7] at a center-of-mass energy of $7 \mathrm{TeV}$ using data corresponding to an integrated luminosity of 5 $\mathrm{fb}^{-1}$. This study considers events with three jets having the highest transverse momenta. The cross section is determined as a function of the invariant mass of the three jets as well as in terms of the maximum rapidity of the jets. A comparison between the measurement and the prediction from perturbative QCD at NLO is performed (Figure 6 (a)) where it can be seen that data and theory calculations with different PDF sets are in agreement within their uncertainties. Some deviations are observed for ABM11 PDF set.

\subsection{Inclusive Multijet production at $\sqrt{s}=7 \mathrm{TeV}$}

CMS publication [8] studies distributions of topological observables in inclusive three- and 
Figure 4: Dijet cross section as a function of dijet azimuthal decorrelation variable, in jet $\mathrm{p}_{T}$ slices, normalized to total dijet cross setion

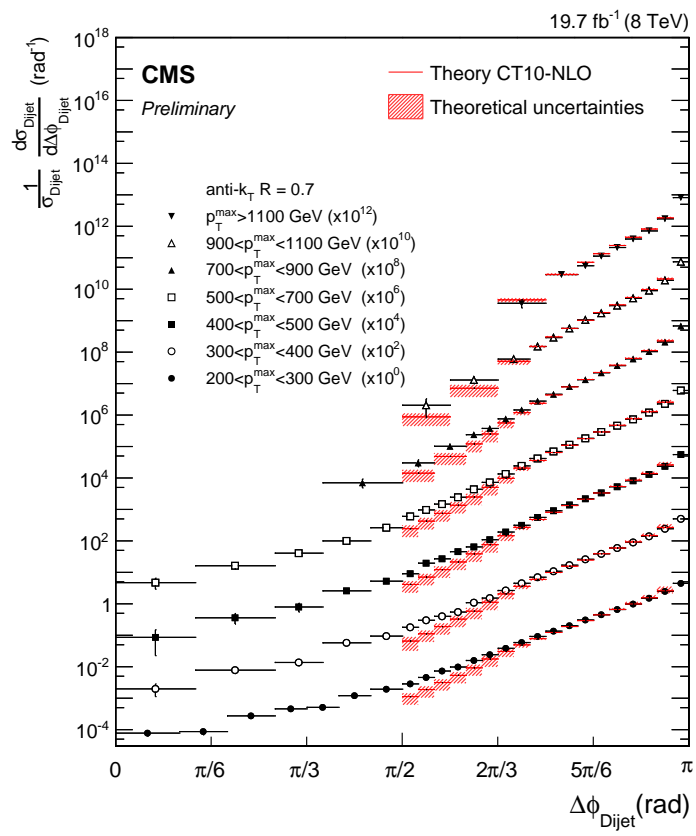

Figure 5: (a) Comparison between data to theories arising from different PDF sets (b) Comparison between data and various Monte Carlo event generators
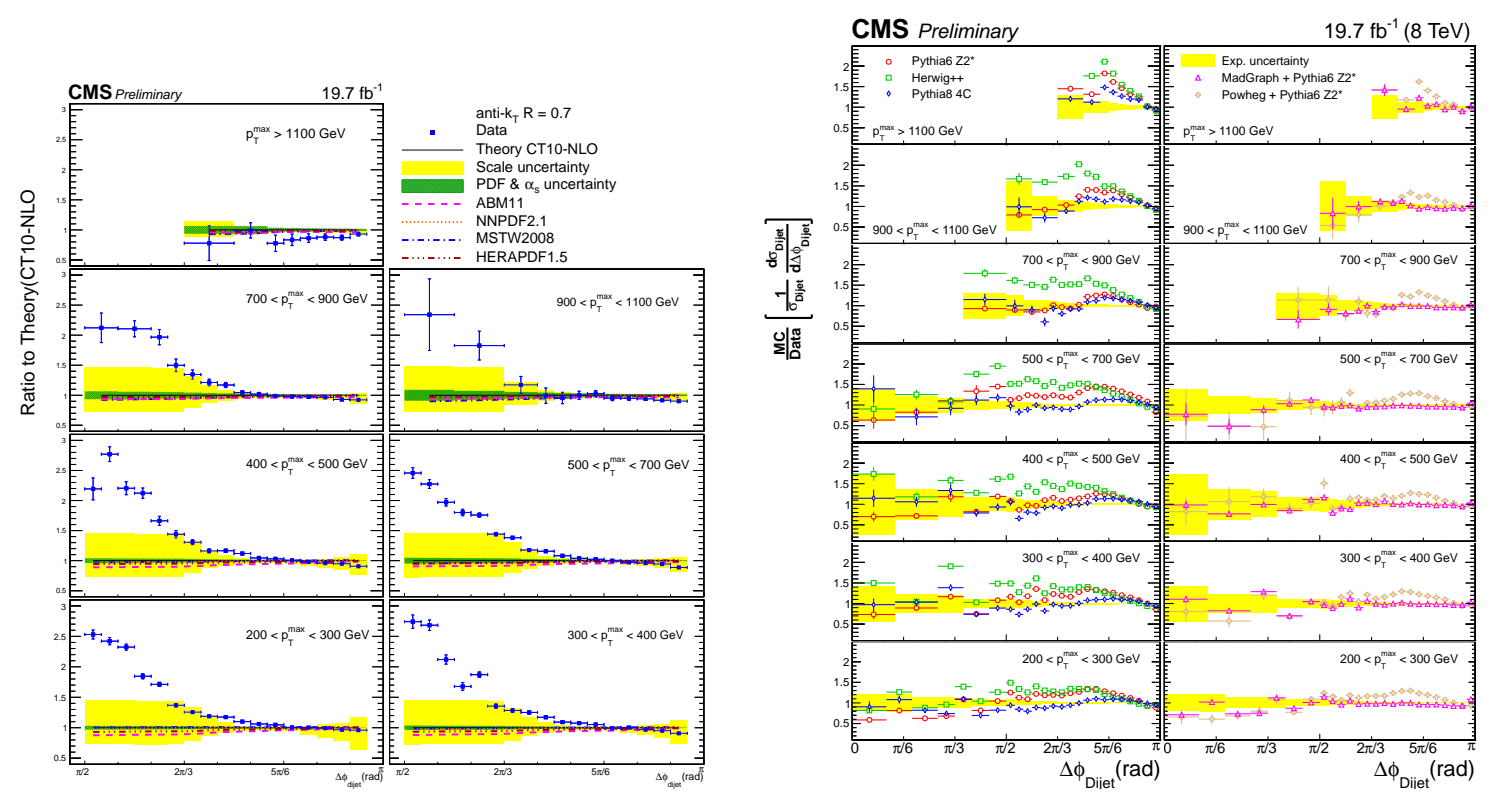
Figure 6: (a) Comparison of data to theory where theoretical calculations are performed with different PDF sets (b) Comparison of data to Monte Carlo predictions for $\mathrm{x}_{3}$ variable


four-jet events produced in pp collisions at a center-of-mass energy of $7 \mathrm{TeV}$ corresponding to a luminosity of $5 \mathrm{fb}^{-1}$. The distributions that are unfolded to nullify the detector effects, are compared with several event generators at leading order. Among them, MADGRAPH showered with PYTHIA6 indicates the overall best agreement with the data. Among studied variables the comparison between unfolded scaled leading-jet energy variable $\left(\mathrm{x}_{3}\right)$ to predictions coming from several event generators is depicted in Figure 6 (b).

\subsection{Summary}

The results presented in these proceedings provide a deep insight to understand perturbative QCD and non-perturbative QCD physics in LHC as it approaches higher energy. Results can be further utilized in improving models in simulation, constraining PDFs, determining strong coupling constant.

\section{References}

[1] S. Chatrchyan et al. [CMS Collaboration], JINST 3, S08004 (2008).

[2] CMS Collaboration, CMS-PAS-FSQ-12-031.

[3] CMS Collaboration, CMS-PAS-SMP-12-012.

[4] M. Cacciari, G. P. Salam and G. Soyez, JHEP 0804, 063 (2008) [arXiv:0802.1189 [hep-ph]].

[5] CMS Collaboration, CMS-PAS-SMP-14-017. 
[6] CMS Collaboration, CMS-PAS-SMP-14-015.

[7] V. Khachatryan et al. [CMS Collaboration], Eur. Phys. J. C 75, no. 5, 186 (2015) [arXiv:1412.1633 [hep-ex]].

[8] V. Khachatryan et al. [CMS Collaboration], Eur. Phys. J. C 75, no. 7, 302 (2015) [arXiv:1502.04785 [hep-ex]]. 